\title{
Detection and Identification System of Bacteria and Bacterial Endotoxin Based on Raman Spectroscopy
}

\author{
Muhammad Elsayeh \\ Systems and Biomedical Engineering \\ Faculty of Engineering- Cairo University \\ Giza, Egypt
}

\author{
Ahmed H.Kandil \\ Systems and Biomedical Engineering \\ Faculty of Engineering- Cairo University \\ Giza, Egypt
}

\begin{abstract}
Sepsis is a global health problem that causes risk of death. In the developing world, about 60 to $80 \%$ of death cases are caused by Sepsis. Rapid methods for detecting its causes, represent one of the major factors that may reduce Sepsis risks. Such methods can provide microbial detection and identification which is critical to determine the right treatment for the patient. Microbial and Pyrogen detection is important for quality control system to ensure the absence of pathogens and Pyrogens in the manufacturing of both medical and food products. Raman spectroscopes represent a $q$ uick and accurate identification and detection method, for bacteria and bacterial endotoxin, which this plays an important role in delivering high quality biomedical products using the power of Raman spectroscopy. It is a rapid method for chemical structure detection that can be used in identifying and classifying bacteria and bacterial endotoxin. Such a method acts as a solution for time and cost effective quality control procedures.

This work presents an automatic system based on Raman spectroscopy to detect and identify bacteria and bacterial endotoxin. It uses the frequency properties of Raman scattering through the interaction between organic materials and electromagnetic waves. The scattered intensities are measured and wave number converted into frequency, then the cepstral coefficients are extracted for both the detection and identification. The methodology depends on normalization of Fourier transformed cepstral signal to extract their classification features. Experiments' results proved effective identification and detection of bacteria and bacterial endotoxin even with concentrations as low as 0.0003 Endotoxin unit $(\mathrm{EU}) / \mathrm{ml}$ and 1 Colony Forming Unit (CFU)/ml using signal processing based enhancement technique.
\end{abstract}

Keywords-Rapid Microbial Detection; Rapid Pyrogen Detection; Microwave Spectroscopy; Dielectric Spectroscopy; Ultra Wide Band; Cepstral Analysis; Raman Spectroscopy

\section{INTRODUCTION}

Endotoxaemia and Sepsis are the leading cause of death in surgeries [1][2]. One of its causes is the absence of an automatic system for bacteria detection and identification that provides a rapid method for bacteria and bacterial endotoxin detection to choose the suitable clinical therapeutic procedures. Such a system may represent essential tool for a quality control system in pharmaceutical industry of intravenous injection products [3][4].

Endotoxins are Lipopolysaccharide (LPS) Pyrogens produced by negative gram bacteria outer cell walls5.
There are several methods for bacteria identification and classification such as gram staining, culturing and biochemical methods. Another known method such as polymerase chain reaction used to amplify short DNA fragments (Primers) that recognize sequences of genes that encode essential molecule. In case of bacteria detection and identification, the PCR method depends on the primers of DNA sequences of bacteria genes [6]. PCR needs sophisticated equipment, several components and reagents. PCR testing takes greater than 15 minutes [6].

Another drawback is the difficulty to identify and detect mixed containment. One of the new and instrumental based rapid method is the adenosine triphosphate (ATP) bioluminescence. It depends on special sample preparation utilizes specific enzyme combination and surfactant. The enzyme breaks down microbial ATP and produce visible light which measured the microbial presence. One of main drawback is the non-microbial ATP reaction and false positive indication. It is time consuming method requiring 24-48 hours to be completed. There are many other instrumental based rapid methods for microbial detection [7]. However, they are rapid, they have drawbacks such as time consuming, lower specify, false identification and quantification.

On the other hand, there are several endotoxins' assays such as Thiobarbituric acid -assay, Rabbit Pyrogen Test(RPT), Human blood test , Endotoxin Activity Assay and the well-known (gel clotting or photometric) Limulus Amebocyte Lysate (LAL) assay [1][8]. Different methods and instrumentation, used in detection of endotoxin, already exist such as Capillary electrophoresis, Laser Induced Fluorescence (CE-LIF), Gas chromatography -Mass Spectroscopy (GCMS), Matrix - Assisted Laser Desorption Ionization -Time of Flight Mass spectroscopy(MALDI-TOF-MS), Ion trap mass spectroscopy and Fourier Transform Ion Cyclotron Resonance Mass Spectroscopy[8-10]. However, since using the above mentioned instrumental methods, is very artful and complicated. This due to the fact that as these methods need samples' preparations and derivatization as preparatory step for instrument to detect the LPS. As a result, these methods are time consuming which is not preferred especially in case of severe sepsis that cause death in less than an hour. Moreover, they need complex procedures to ensure accurate measurements and have expensive implementations.

The main objective of this research is to establish an alternative accurate method for rapid bacterial and bacterial 
endotoxin detection and identification with low running cost. Towards the progress in rapid microbial method development, vibrational spectroscopes took a contribution in developing new automatic system based on the Surface Enhanced Raman Spectroscopy (SERS). SERS is in Raman Spectroscopy to enhance the Raman scatter intensity of biological sample which is very weak as it suffers from high fluorescence background due to presence of macromolecules which is fluorophore in nature. The technique mainly depends on plasmon resonance [11][12].

Raman Spectroscopy is a vibrational spectroscopy which characterizes the material based on vibrational frequencies resulting from inelastic scatters of laser source's polarization effect. Vibrational and rotational spectroscopy are linked to the main electromagnetic wave interaction properties which is the dielectric mechanisms or polarization effects(Electronic and atomic Polarization, Dipole and ionic relaxation)[13][14].Since the materials containing charged particles produce secondary fields when it comes in contact with electric or magnetic fields. They result in conduction, polarization, or magnetization of the particles in that material. Polarization of the particles forces the material to act as a dielectric [14][15]. The permittivity is determined by dielectric material's ability to polarize its particles under the influence of an electric field [13][14]. The polarization effect or dielectric mechanism is linked to the material's interaction properties under study of the electromagnetic waves, especially electric field components of the wave. The vibrational mode of the molecule depends on the frequency of the applied field. The vibrational motion of the molecules induces a displacement in molecule equilibrium position, called electric displacement and can be expressed by equation (1)

$$
D(\omega)=\varepsilon_{0} E(\omega)+P
$$

Where, $\omega$ is the angular frequency $2 \pi f, E(\omega)$ is the electric field of the microwave relative to oscillation frequency, $P$ is the Polarization density corresponding to dipole moment density expressed by equation (2)

$$
P=\alpha E
$$

So the displacement occurring is related to the applied electric filed which induced by the dipole moment, where $\alpha$ is the molecular polarizability which is a material property that depend on the material structure and bond nature. The polarizability has direct link with material dielectric properties as expressed in equation (3) and (4)

$$
\alpha=\chi_{e} \varepsilon_{0}
$$

Where $\chi_{e}$ is the electric susceptibility of the material and can be expressed as equation (4)

$$
\chi_{e}=\varepsilon^{*}(\omega)-1
$$

Where, $\varepsilon^{*}(\omega)$ is the complex permittivity relative to each frequency variation. According to equation (3) and (4), $\alpha$ can be expressed as follow in equation (5)

$$
\alpha=\left(\varepsilon^{*}(\omega)-1\right) \varepsilon_{0}
$$

By substituting in equation (2) using equation (5), we can express the dipole moment in terms of complex permittivity and electric field relative to frequency variation in equation (6)

$$
P(\omega)=\left(\varepsilon^{*}(\omega)-1\right) \varepsilon_{0} E(\omega)
$$

In case of electronic and atomic polarization the induced dipole moment mechanism will be related to the exciting and radiated photons. Accordingly, dipole moment can be represented in different form as defined in equation (7)

$$
\begin{aligned}
& P=\alpha_{o} E_{o} \cos \left(2 \pi f_{o} t\right)+\frac{1}{2}\left(\frac{\partial \alpha}{\partial q}\right) q_{o} f_{o}\left[\cos \left(2 \pi\left(f_{0}+f_{m}\right) t\right)+\right. \\
& \left.\cos \left(2 \pi\left(f_{0}-f_{m}\right) t\right)\right]
\end{aligned}
$$

The equation represent oscillating dipole which radiates photons with three different frequencies $f_{0}$ (elastic scattering), $f_{0}+f_{m}$ (inelastic scattering with shorter wave length) and $f_{0}-f_{m}$ (inelastic scattering with longer wave length). Where $f_{0}$ is the frequency from the vibrating molecules of the sample scattered is, $f_{m}$ is the frequency of the vibrational mode and $q_{o}$ is the vibrational amplitudes.

The polarizability should be changed with vibrational displacement $q$, the change of Raman intensity is proportional to the square of the rate change of the polarizabiltiy with respect to change of the displacement $\left(\frac{\partial \alpha}{\partial q}\right)_{0}^{2}$. In equation (5) the polarizability is related to dielectric properties of the material, so the intensity amplitude will change depending on the molecular composition of the sample.

The different dielectric mechanism can be cross linked with material properties and its interaction with the excitation source. As the scattering parameters are in the scope of this study, we will find a similarity of the analysis developed in the authors' previous work [14] however the excitation source is different. The paper is organized as follows, the materials and methods are described in section two. The results and discussion are included in section three. The conclusion of this work is presented in section four.

\section{MATERIALS AND METHODS}

\section{A. Experiment Setup}

The process steps and setup can be defined as shown in process diagram figure (1). The excitation source is based on a Raman Analyzer from Thermo Scientific. The Laser excitation source of $785 \mathrm{~nm}$ and output power of $250 \mathrm{~mW}$. The spectral range from 250 to $2875 \mathrm{~cm}-1$. The sample is placed in contact with Raman analyzer focal lens about $18 \mathrm{~mm}$ to focal plane. The actual measuring distance is $4.75 \mathrm{~mm}$ from outer surface of the sample test tube. The test tube is made of borosilicate glass of $16 \mathrm{~mm}$ in diameter and $125 \mathrm{~mm}$ in height. The test tube has wall thickness of $1.8 \mathrm{~mm}$.

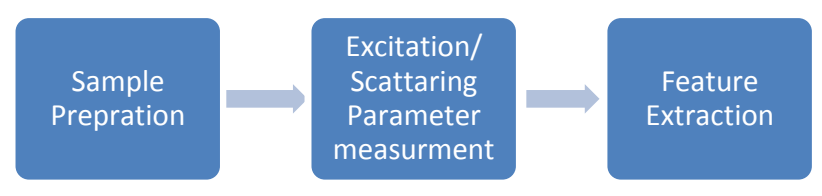

Fig. 1. Method Process diagram 


\section{B. Samples Preperations and Measutements}

The reference samples were consisted of two water types (LAL Reagent water, Controlled distilled water free of microbial contamination and of less than $0.25 \mathrm{EU} / \mathrm{ml}$ ). The samples under test were divided into endotoxin containment and microbial containment. The serial dilutions of different concentrations were prepared by a control spike which is a lipopolysaccharide from E.coli055:B5 as certified by Charles River Endosafe ${ }^{\circledR}$ as shown in Table (1). Bacteria culturing were used to prepare different bacteria containment, we used Nutrient broth and agar slants to prepare lyophilized cultures of (Kocuria rhizophila (Micrococcus luteus) ATCC 9341, Pseudomonas aeruginosa ATCC 9027, Staphylococcus aureus ATCC 6538P,Escherichia coli ATCC 25922 and Staphylococcus haemolyticus ATCC 29970D-5.

In Raman Spectroscopy experiments, the data collected were Raman scatter signals which represented in Raman amplitude and Raman shift. Raman shift is the change in the frequency between the incident and emitted light as in equation (8)

$$
\Delta v\left(\mathrm{~cm}^{-1}\right)=\left(\frac{1}{\lambda_{\text {incident }}}-\frac{1}{\lambda_{\text {scattered }}}\right) \times 10^{-7}
$$

TABLE I. CONTAINMENT SAMPLES CONCENTRATIONS

\begin{tabular}{|c|c|c|c|}
\hline Samples & $\begin{array}{l}\text { Concentration } \\
\text { EU/ml }\end{array}$ & Samples & $\begin{array}{l}\text { Concentration } \\
\text { EU/ml }\end{array}$ \\
\hline 1 & 0.0003 & 2 & 0.003 \\
\hline 3 & 0.03 & 4 & 0.06 \\
\hline 5 & 0.125 & 6 & 0.25 \\
\hline 7 & 0.5 & 8 & 2.5 \\
\hline 9 & 5 & 10 & 50 \\
\hline 11 & 100 & 12 & 1000 \\
\hline 13 & 10000 & 14 & 100000 \\
\hline 15 & Reference Sample \\
\hline
\end{tabular}

\section{Feature Extraction Algorithm}

The algorithm depends on the nature of the output signal. The signal is defined by Raman shift and intensity. We convert the represented wave number into frequency as defined by equation (9) and apply the Cepstral transform for the signal by using equation (10).

The main goal is to find the variance between reference and contaminated sample in terms of specific signature of bacterial endotoxin and bacteria cells. Cepstral analysis algorithm is a well-known algorithm in speech recognition applications and analysis of radar signal returns applications [17]. The Cepstrum is defined as the inverse Fourier transform of the log-magnitude Fourier Spectrum [18]. Windowed liftring $w(n)$ is applied on the Cepstrum to eliminate the highest and lowest quefrencies by using equation (11). Then, a final signature can be obtained by taking the Fourier transform of the sample signal and normalizing it by the reference signal on its Frequency domain form.

$$
\begin{gathered}
f=c \times \Delta v\left(\mathrm{~cm}^{-1}\right) \\
C_{S_{\text {Raman }}}(Q)=F^{-1}\left\{20 \times \log \left(S_{\text {Raman }}(f)\right)\right\} \\
w(n)=\left\{\begin{array}{cc}
0.5 & 1 \leq n \leq 2 \\
1 & 2<n<N-20 \\
0.5 & N-20 \leq n \leq N
\end{array}\right.
\end{gathered}
$$

The detection signature can be calculated as follow

$$
S_{\text {detection }}=\frac{F\left\{C_{\left.S_{\text {Raman (containment })}(Q) * w(n)\right\}}(Q) * w(n)\right\}}{F\left\{C_{S_{\text {Raman }(\text { Ref })}}(Q) * w\right.}
$$

\section{RESULTS AND DISCUSSION}

Figure (2) shows the raw Raman spectrum for the lowest $(0.0003 \mathrm{EU} / \mathrm{ml})$ and highest endotoxin concentration $(100,000$ $\mathrm{EU} / \mathrm{ml}$ ) against the same Pyrogen free reference sample. It shows the variance occur to laser scattered waves due to the change in material properties as result of contamination with different concentration. The same waveform response is detected due to the strong scattering from Borosilicate glass. A weak response was detected this was shown as an absence of specific wave form for water and endotoxin at their known wave number assignments. Water cannot be detected due to device's spectral range limitation. Water Raman spectra range is from $\left(3000-3800 \mathrm{~cm}^{-1}\right)$. Also Lipid-A potion in LPS cannot be detected due to spectral range limitation which have strong response at $2934 \mathrm{~cm}^{-1}$.

Figure (3),(4) are the raw Raman spectra of the two positive gram (staphylococcus haemolyticus and micrococcus luteus) bacteria and two negative gram bacteria (Pseudomonas aeruginosa and Escherichia coli) inside distilled water. The distilled water has endotoxin concentration $<0.25 \mathrm{EU} / \mathrm{ml}$. The results show no significant difference in wave form or intensity which show weak scatter and spectral information for bacteria samples.

The signals showed in these figures contain hidden endotoxin, bacteria Raman scattered data.

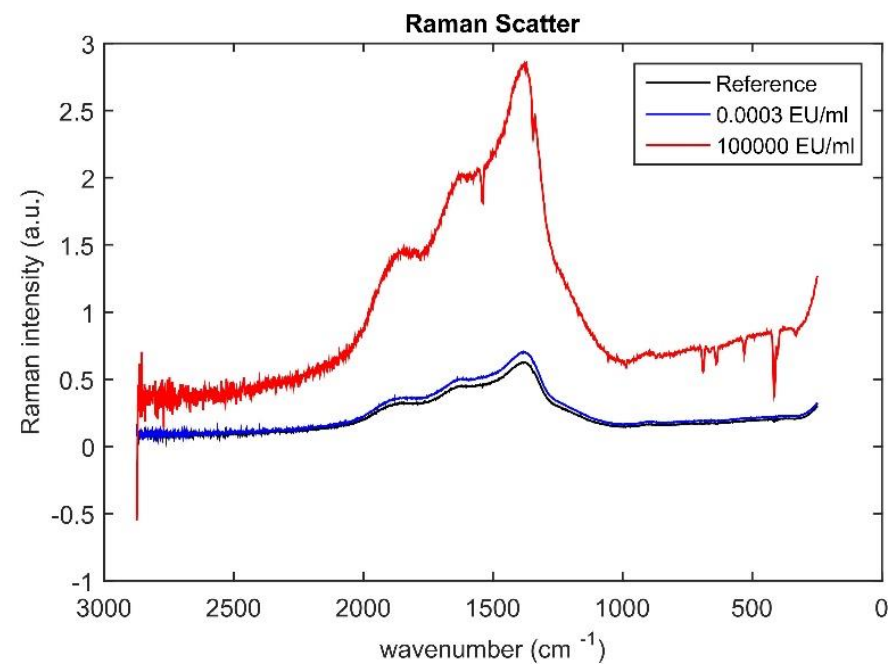

Fig. 2. Raman Spectrum for lowest and highest endotoxin concentration against reference pyrogen free distilled water 


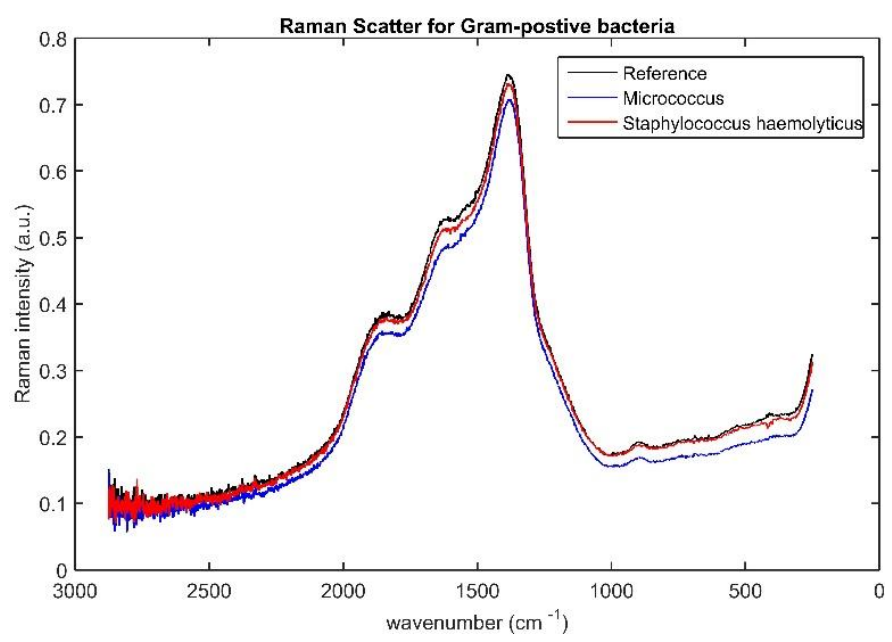

Fig. 3. Raman Spectrum for different mixture of gram Positive bacteria and distilled water with endotoxin less than $0.25 \mathrm{EU} / \mathrm{ml}$

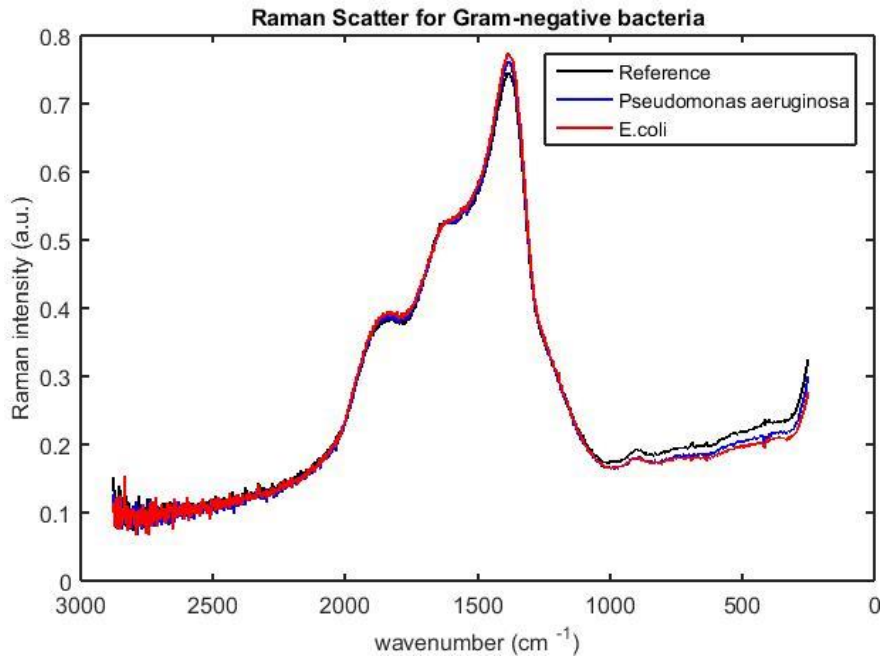

Fig. 4. Raman Spectrum for different gram negative bacteria and distilled water with endotoxin less than $0.25 \mathrm{EU} / \mathrm{ml}$

Power spectrum representation of Raman spectrum show the specific existence of endotoxin which can be used in the bacteria classification as shown in figure $(5 \mathrm{a})$. The endotoxin samples can be discriminated from the gram negative bacteria by the amplitude value and the specified frequency in the band region from 4 to $5 \mathrm{GHz}$. This can be correlated with the endotoxin material specific response in frequency bands (4 - 5 $\mathrm{GHz}$ ) of microwave scattering parameter $\mathrm{S} 11$ as shown in figure (5b and 5c) [14].This frequency band is related to actual wave number assignment known for LPS which is $\left(1400-1500 \mathrm{~cm}^{-1}\right.$ equivalent to $\left.4-4.5 \mathrm{GHz}\right)$. These wave numbers band cover carbohydrate, lipid and protein constitutes of gram negative outer membrane and cell wall, viz, lipopolysaccaraides, lipoproteins, phospholipids, proteins and porins [19]. Wave number band $\left(1500-1600 \mathrm{~cm}^{-1}\right.$ equivalent to $4.5-5 \mathrm{GHz}$ ) cover carotenoid and nucleic acids [19]. The technique was challenged to determine if it can detect two mixtures of the same class of bacteria containment. In Figure (6), the output signal captured for the mixture is a result of mean value of the two main signals which represent the signature of each individual bacteria.

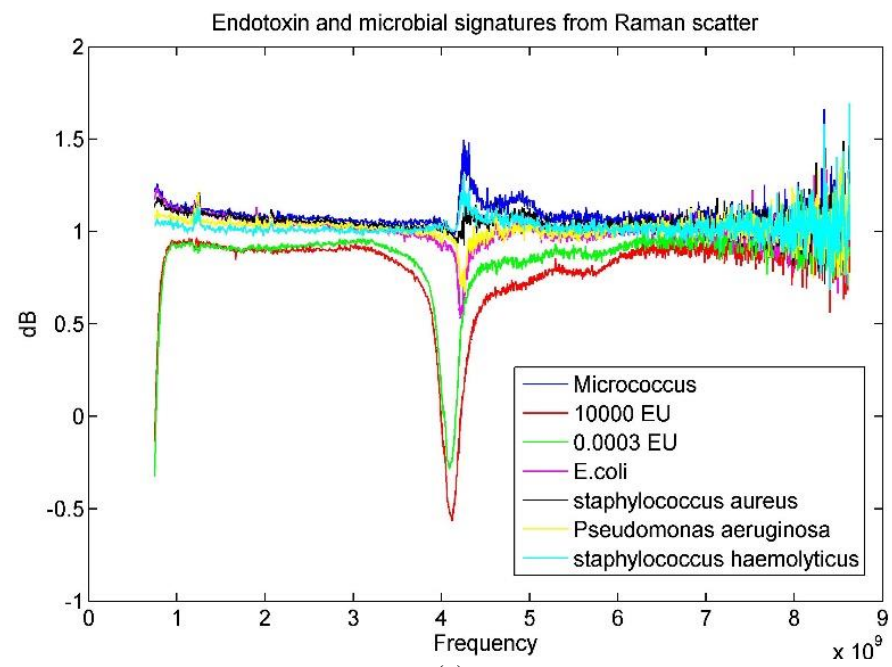

(a)

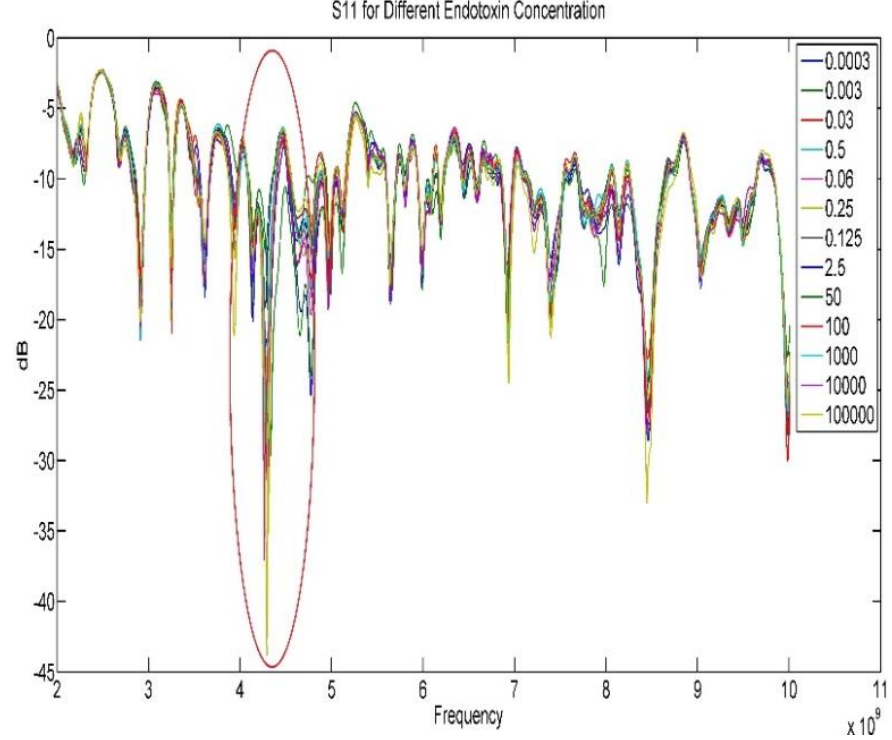

(b)

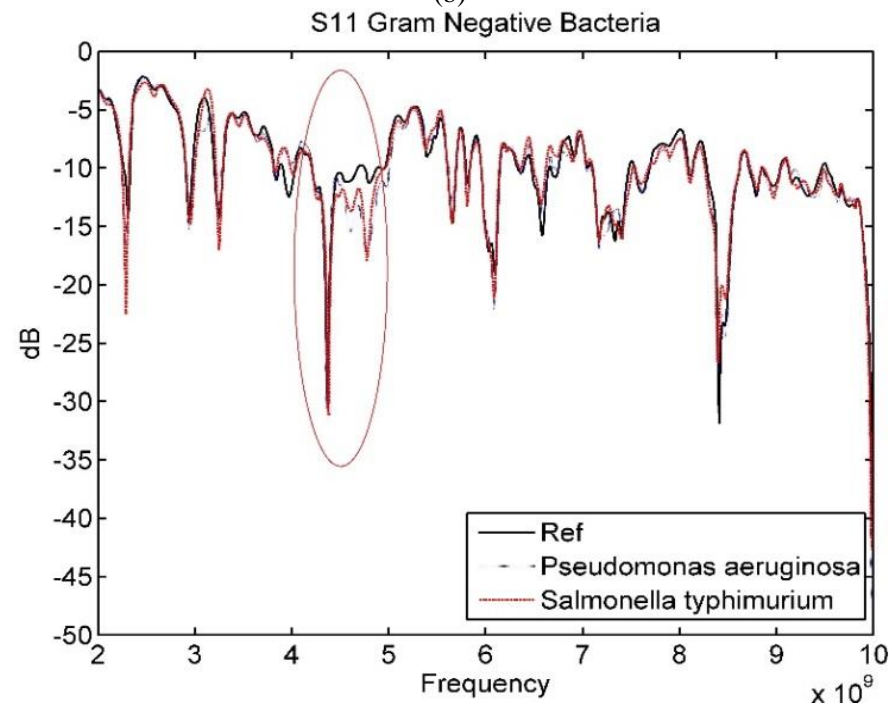

(c)

Fig. 5. a) Normalized filtered Raman power spectrum for different bacteria species and different endotoxin concentration. b) S11 signal for Different Endotoxin concentration, the circular annotation shows the specific wave 
form and response from 4 to $5 \mathrm{GHz}$ bandwidth [14]. c) S11 signal for different gram negative bacteria contaminant a distilled water sample with endotoxin less than $0.25 \mathrm{EU} / \mathrm{ml}[14]$

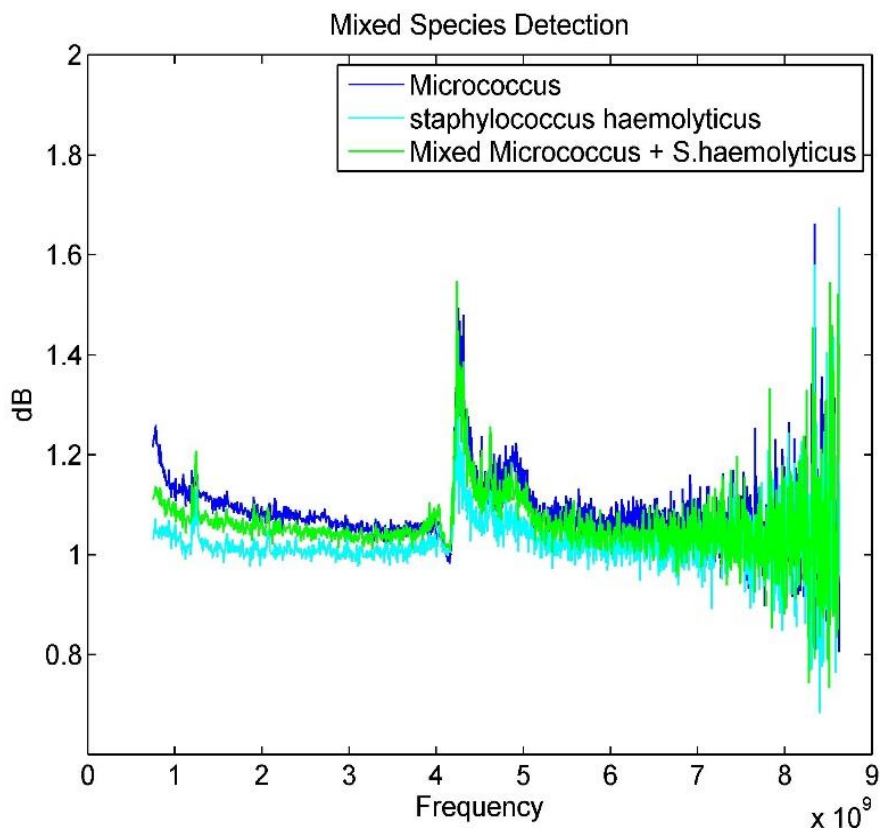

Fig. 6. Normalized filtered Raman power spectrum for different mixture of gram positive bacteria species against individual species

\section{CONCLUSION}

A new technique for Raman enhancement using signal processing to identify and detect bacteria and bacterial endotoxin. It shows good sensitivity in LPS feature extraction and detection. It can be used as low cost, simple and rapid method for microbial and pyrogen detection applications. Also, it can be used for different bacteria identification and classification system. The method has low processing time less than $1 \mathrm{~min}$.

We have proven that the frequency component of the lipopolysaccharide Raman scattered as same as the Ultra Wide Band (UWB) reflection scattered parameter measured in our previous work [14]. Although they are totally different electromagnetic excitation source but they have the same effect of standing wave caused by the interaction between the incident and scattered field that can be described in terms of reflection coefficient which, in turn, is related to the backscatter efficiency and similarities. In addition, we have offered solution for detection of bacteria mixture from different species in same medium.

\section{ACKNOWLEDGMENT}

We need to thank GalxoSmithKline-Giza site in Egypt, Chemistry analytical quality control Laboratory: Dr.Ahmed Ramadan and his team, Microbiology laboratory : Dr. Manal Aref and her team and Dr. Odette Boulos, head of quality for offering microbial and Pyrogen samples preparation and usage of Raman analyzer on their work place.

\section{REFERENCES}

[1] B. Eisele, M. Lamy, L.G. Thijs, H.O. Keinecke, H.P. Schuster, F.R. Matthias, E. Fourrier, Fourrier, H. Fourrier, U. Delvos, Antithrombin III in patients with severe sepsis, A randomized, placebo-controlled, double-blind multicenter trial plus a meta-analysis on all randomized, placebo-controlled, double-blind trials with antithrombin III in severe sepsis, Intensive Care Medicine, 24 (7) (1998) 663-672.

[2] E. Esteban, R. Ferrer, L. Alsina, A. Artigas, " Immunomodulation in sepsis: the role of endotoxin removal by polymyxin B-immobilized cartridge, "Mediators Inflamm; vol.2013:507539, (2013).

[3] FDA, "Guidance for Industry: Pyrogen and Endotoxins Testing: Questions and Answers", http://www.fda.gov/Drugs/GuidanceComplianceRegulatoryInformation/ Guidances/ucm314718.htm, (2012).

[4] M. Daneshian, A. Guenther, A. Wendel, T. Hartung, S. von Aulock, In vitro pyrogen test for toxic or immunomodulatory drugs, Journal of Immunological Methods, 313 (2006) 169-175.

[5] E.T. Rietschel, T. Kirikae, F.U. Schade, U. Mamat, G. Schmidt, H. Lippnow, A.J. Ulmer, U. Zähringer, U. Seydel, F. di Padova, M. Schreier, H. Brade, "Bacterial endotoxin: molecular relationships of structure to activity and function", FASEB J. 8, 217-225, (1994).

[6] A.K. Järvinen, S. Laakso , P. Piiparinen, A. Aittakorpi, M. Lindfors, L. Huopaniemi, H. Piiparinen, and M. Mäki,'Rapid identification of bacterial pathogens using a PCR- and microarray-based assay" BMC Microbiology 2009, 9:161,http://www.biomedcentral.com/14712180/9/161, (2009).

[7] L. Jimenez, "Molecular Diagnosis of Microbial Contamination in Cosmetic and Pharmaceutical Products: A Review", Journal of AOAC international Vo.84, No.3, (2001).

[8] A. Bui, A. Kilár, Á. Dörnyei, V. Poór, K. Kovács, B. Kocsis, F. Kilár,” Carbohydrate composition of endotoxins from R-type isogenic mutants of Shigella sonnei studied by capillary electrophoresis and GC-MS", Croatica Chemica Acta ,84 (3) (2011) 393-398.

[9] A. Bui, B. Kocsis, and F. Kilár, "Methodology to label mixed carbohydrate components by APTS", Journal of Biochemical and Biophysical Methods, 70 (6) (2008) 1313-1316.

[10] A. Kilár, Á. Dörnyei, A. Bui, Z. Szabó, B. Kocsis, and F. Kilár, "Structural variability of endotoxins from R-type isogenic mutants of Shigella sonnei", Journal of Mass Spectrometry, 46 (1) (2011) 61-70.

[11] K. Kneipp, A.S Haka, H. Kneipp, K. Badizadegan, N. Yoshizawa, C. Boone, K.E. Shafer-Peltier, J.T. Motz, R.R. Dasari, and M.S. Feld, "Surface-enhanced Raman spectroscopy in singleliving cells using gold nanoparticles," AppliedSpectroscopy 56 (2002) 150-154.

[12] A. Campion and P. Kambhampati, "Surface-enhanced Raman scattering," Chemical Society Reviews 27, (1998) 241-250.

[13] Jovan Mijovic and Benjamin D.Fitz, "Dielectric Spectroscopy of reactive Polymers", Department of chemical Engineering, Chemistrry and Materials Science, Polytechnic University, six Metrotech Center, Brooklyn, NY 11201, Novocontrol Application Note Dielectrics 2, 1998.

[14] M.Elsayeh, A.H.Kandil, “ Ultra Wide Band Based Quantitative and Qualitative Method for Bacterila Endotoxin Detection", Journal of Microwave Power and Electromagnetic Energy 49 (1) (2015) pp. 11- 20.

[15] N.N. Rao, "Elements of Engineering Electromagnetics", 2nd ed. Englewood Cliffs, New Jersey: Prentice-Hall, Inc, (1987).

[16] A. M. Nicolson, G. F. Ross, "Measurement of the Intrinsic Properties of Materials by Time Domain Techniques", Instrumentation and Measurement, Vol. 19, (1970), pp.377-382,. doi: 10.1109/TIM.1970.4313932,.

[17] A.M. Noll, "Cepstrum Pitch Determination," Journal of the Acoustical Society of America, Vol. 41, No. 2 (1967) pp. 293-309.

[18] J.G. Proakis, D. G. Manolakis,"Digital signal processing”. Pearson Prentice Hall. ISBN 978-0-13-187374-2, (2007).

[19] J.R. Goodwin, L.M. Hafner, P.M. Fredericks, “ Raman Spectroscopy of the Heterogeneity of Microcolonies of a Pigmented Bacterium", Journal of Raman Spectroscopy 37 (2006) P.932-936. 